\title{
Massive star evolution in the Small Magellanic Cloud
}

\author{
Daniel J. Lennon \\ The Isaac Newton Group of Telescopes, Apartado de Correos 321, \\ E-38700, Santa Cruz de La Palma, Canary Islands, España
}

\begin{abstract}
We discuss abundances for eight early B-type giant/supergiant stars in the SMC cluster NGC 330. All are nitrogen rich with an abundance approximately $1.3 \mathrm{dex}$ higher than an SMC main-sequence field. Given the number of B-type stars with low rotational projected velocities in NGC 330 (all our targets have $v \sin i<50 \mathrm{~km} \mathrm{~s}^{-1}$ ), we suggest that it is unlikely that the stars in our sample are seen almost pole-on, but rather that they are intrinsically slow rotators. Comparing these results with the predictions of stellar evolution models including the effects of rotationally induced mixing, we conclude that while the abundance patterns may indeed be reproduced, those models with initially large rotational velocities do not reproduce the observed range of effective temperatures of our sample, nor the currently observed rotational velocities. Binary models may be able to produce stars in the observed temperature range and provide a promising alternative to single star models for explaining the observations. We also discuss the clear need for stellar evolution calculations employing the correct chemical mix of carbon, nitrogen and oxygen for the SMC.
\end{abstract}

\section{Introduction}

Blue and red supergiants (the precursors of core-collapse SN) have long been regarded as key probes of massive star evolution. The number ratio of blue to red $(B / R)$ supergiants in particular, representative of the hot and cool core helium burning phases, are very sensitive to the treatment of convection and mixing. Recently Langer \& Maeder (1995) summarized the situation with special reference to the $B / R$ ratio as a funtion of metalicity and highlighted the fact that all stellar evolution calculations failed to account for the observed trend of increasing $B / R$ with metallicity. They suggested that rotationally induced mixing might supply a solution and indeed the recent calculations by Heger \& Langer (2000), Meynet \& Maeder (2000) and Maeder \& Meynet (2001) would appear to confirm this suggestion. In this paper we compare the predictions of these models with recent observational results, concentrating on the young SMC cluster NGC 330.

\section{NGC 330}

The SMC cluster NGC 330, together with its Galactic analogue, the double cluster $\mathrm{h}+\chi$ Persei, are widely used as calibrators for stellar evolution calculations. The reason for this is that both clusters have significant numbers of blue and red supergiants, in fact the $B / R$ ratio for NGC 330 is often taken as being rep- 


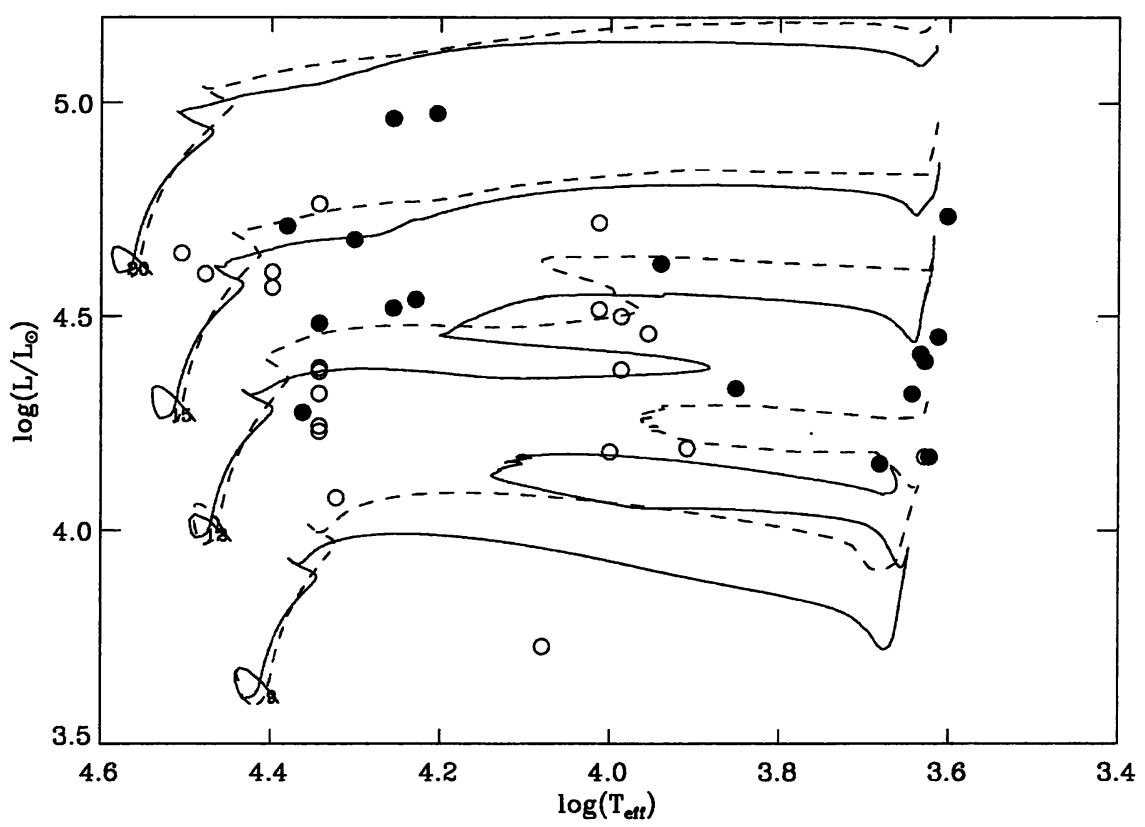

Figure 1. H-R diagram for the brightest stars in NGC 330. Filled circles represent stars for which there are high resolution analyses, while empty circles represent stars for which there is only intermediate or low resolution data. For the former, detailed atmospheric abundances are published, and discussed in the present paper. The evolutionary tracks are from Maeder \& Meynet (2001) for initial rotational velocities of 0 (solid line) and $300 \mathrm{~km} \mathrm{~s}^{-1}$ (dashed line), assuming $Z=0.004$ and initial masses of $9,12,15$ and $20 \mathrm{M}_{\odot}$.

resentative of the SMC and thus the low metalicity $(Z=0.004)$ environment. The features which one would like to compare are the distribution of stars in the $\mathrm{H}-\mathrm{R}$ diagram, in particular both the $\mathrm{B} / \mathrm{R}$ ratio and the effective temperatures of these two groups of supergiants, the surface abundances of the stars as they evolve off the main sequence and, for rotating models, the rotational velocities of the stars. Clearly for binary models one needs information on whether or not individual objects are single or multiple. This aspect of the observations is not addressed by the current data. As has been mentioned, the new rotating models are promising in producing the correct $B / R$ ratio and its trend with metalicity. In this paper we concentrate on abundances, effective temperatures and rotational velocities. Vrancken et al. (2000) have already analysed a number of B-type giants and supergiants $\mathrm{h}+\chi$ Persei and concluded that if nitrogen enrichment was present it must be moderate (relative to the initial abundance). NGC 330, having a much lower initial nitrogen abundance, should provide a clearer test of mixing.

The colour magnitude diagram of NGC 330 was first derived by Arp (1959) who found two groups of supergiants, blue and red, well separated from the main sequence turn-off. These two groups of supergiants have since been associated with the core helium burning phases of evolution. The picture is confused some- 


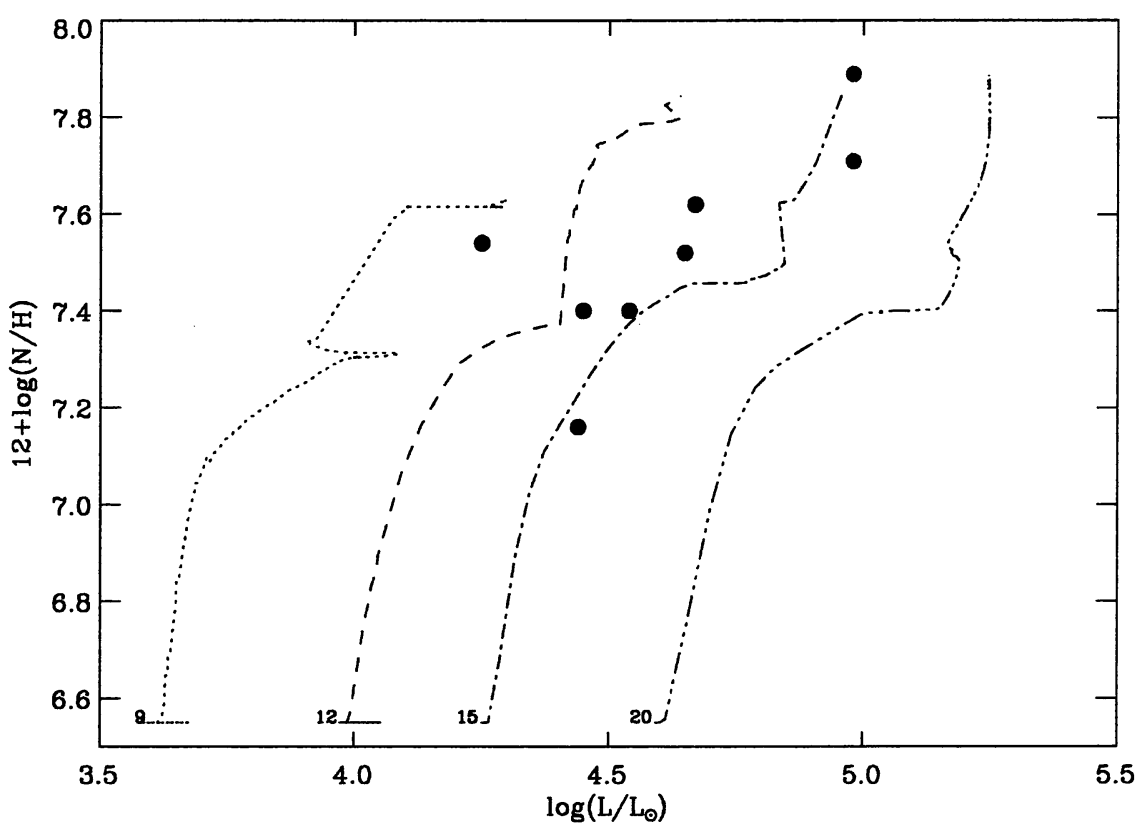

Figure 2. Comparison of observed N abundances for B-type stars in NGC 330 (solid dots, from Lennon et al. 2003) with the predicted values (Maeder \& Meynet 2001, tracks labelled with their initial masses). We have rescaled the $\mathrm{N}$ abundances to an initial $\mathrm{N}$ abundance of $6.55 \mathrm{dex}$ as is appropriate for the SMC. All tracks are for an initial rotational velocity of $300 \mathrm{~km} \mathrm{~s}^{-1}$.

what by the fact that many of the B-type stars at the tip of the supposed main sequence plume are in fact giants, as confirmed by the spectroscopic work of Lennon et al. (1994) and Grebel et al. (1996), who also confirmed that the majority of these B-type stars are Be stars. High resolution observations of B-type giants and supergiants by Lennon et al. $(1996,2003)$, and of K-type supergiants by Hill (1999), have provided additional information on stellar abundances and atmospheric parameters. In this paper we compare these results with the rotational models of Maeder \& Meynet (2001).

\section{Comparison with stellar evolution models}

Using data taken from the references supplied above we can construct an H-R diagram for the brightest stars in NGC330 (Figure 1). We note the following features; a population of B-type giants beyond the end of the main sequence of both the non-rotating and rotating models, two blue straggler stars, two blue supergiants stars which could be consistent with the blue core helium burning phase of the non-rotating 20 solar mass track were it not for the fact that they are strongly nitrogen enriched (discussed below), and some late B-type stars which may be SMC field stars. Conceivably, some of the B-type giants are consistent with fast rotators near the end of their main sequence, and certainly these stars are also nitrogen enriched. In fact, as Lennon et al. (2003) showed, all the B-type stars so far analysed in NGC 330 are nitrogen rich with a mean 


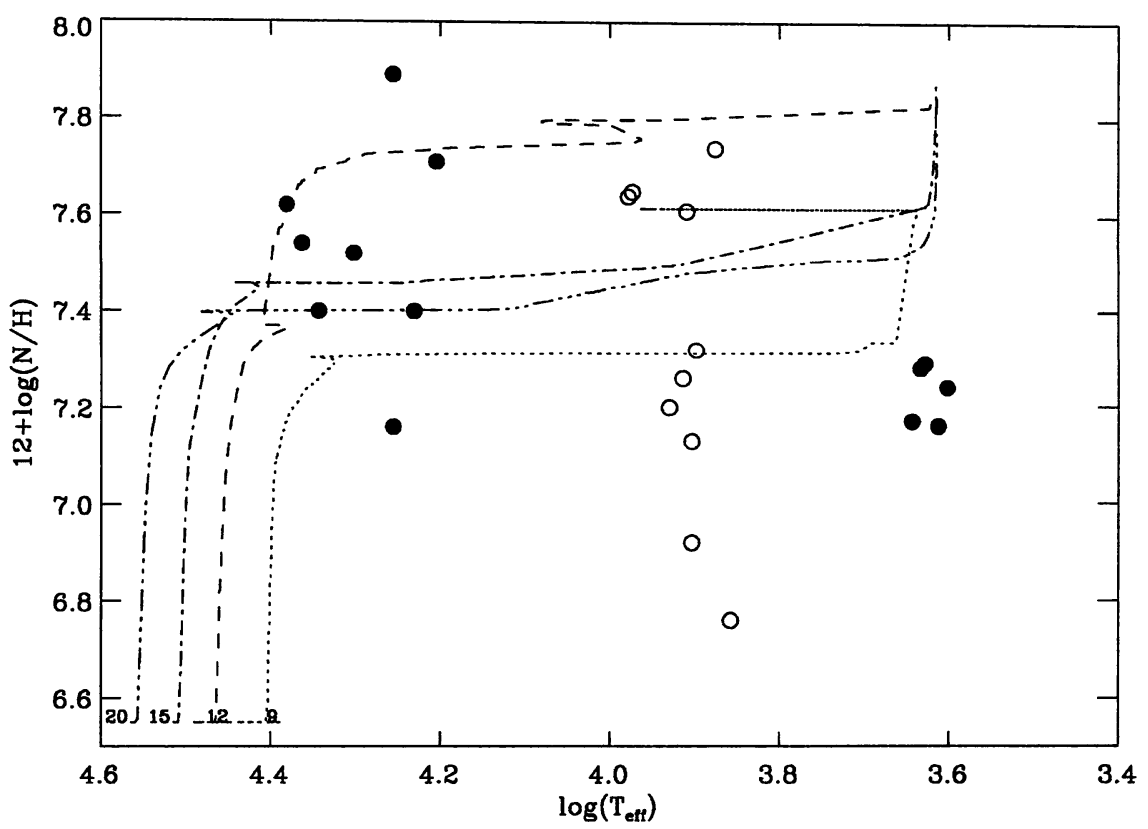

Figure 3. Tracks are as for Figure 2, but abundance is plotted as a function of $T_{\text {eff. }}$. Note that the early B-type stars in the NGC 330 cluster lie just beyond the end of the main sequence between $\log T_{\text {eff }}$ values of 4.2 and 4.4. The A-type supergiants (empty circles) lie in the region of the hot core He-burning phases (depicted by the kinks in the tracks around $\log T_{\text {eff }}=4.0$; note that the $9 \mathrm{M}_{\odot}$ track performs a blue loop).

nitrogen enhancement of more than a factor of 10 . How do these results compare with the predictions of Maeder \& Meynet?

As discussed by Lennon et al. (2003), we must first correct the theoretical results to reflect the very low initial nitrogen abundance of the SMC, we adopt a value of $6.55 \mathrm{dex}$ in the rest of this paper and Figures 2 through 4 . In Figure 2 we compare the nitrogen abundances for the B-type stars in NGC 330 with the stellar evolution tracks with an initial vsini of $300 \mathrm{~km} \mathrm{~s}^{-1}$. While the individual tracks span a range of behaviours (blue loop, no blue loop) the general picture is that these models can clearly reproduce the spread of nitrogen abundances found in the sample of stars. Turning to Figure 3, which compares observations and models in the nitrogen abundance - effective temperature plane we find a serious disagreement for the B-type stars in that they all lie beyond the core hydrogen burning main sequence. They are also too hot for the blue core helium burning phase corresponding to the location of A-type and F-type supergiants. Note that the SMC A-type supergiants from the sample of Venn (1999) have a large spread in nitrogen abundance which is well matched by the upper envelope of the model predictions. The NGC 330 red supergiants analysed by Hill (1999) are clumped rather closely together and the spread is difficult to reconcile with the spread in the B-type stars. Further work on the K-type and A/F-type supergiants in NGC 330 is clearly well worthwhile. 


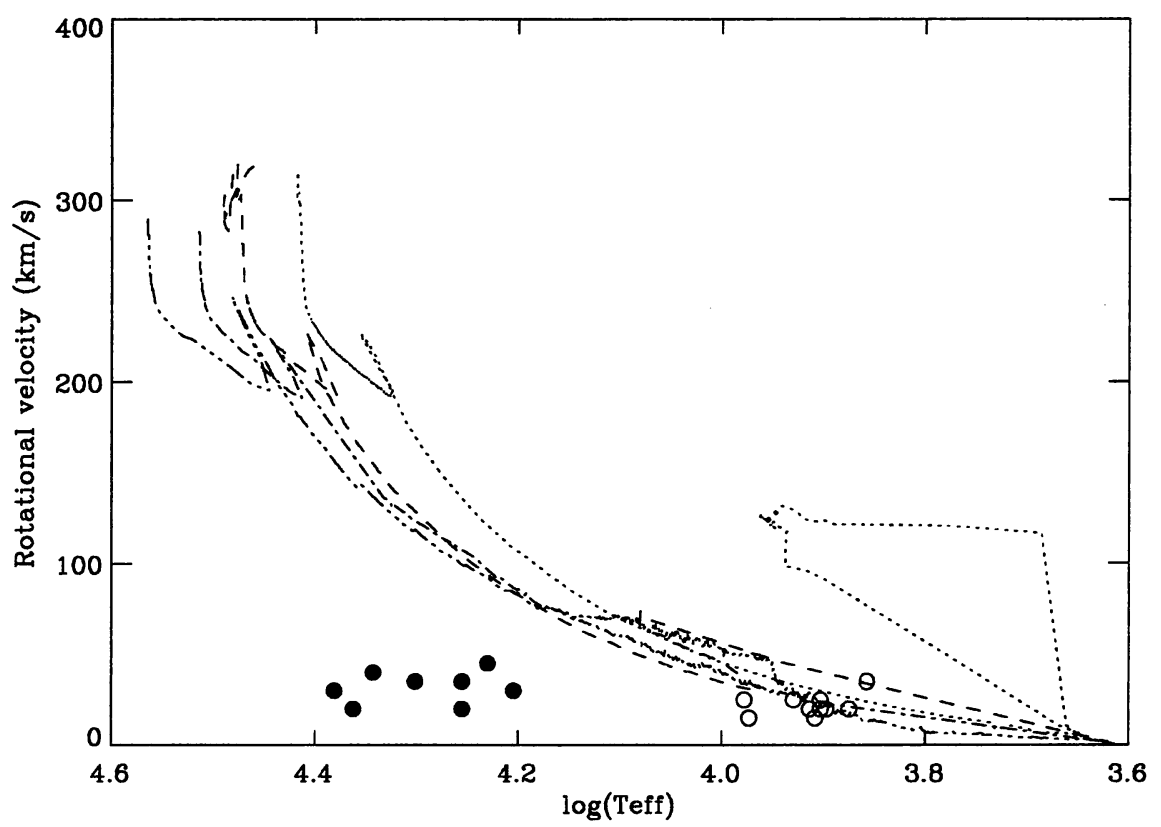

Figure 4. The evolution of the surface rotational velocity is compared with the measured $v \sin i$ values of the B-type stars in NGC 330 from Lennon et al. (2003) and the A-type supergiants from Venn (1999). The stellar evolution tracks are as described in Figures 2 and 3 ; note that the $9 \mathrm{M}_{\odot}$ track exhibits spin-up during its blue loop phase. Note also the large discprepancy for the B-type stars, in particular for those objects near the end of core H-burning.

The models also show the evolution of the surface rotational velocities and in Figure 4 we compare their predictions with the measured values of $v \sin i$. For comparison we also show the values measured by Venn for the A-type supergiants (assuming these are typical of A-type supergiants in NGC 330), the $\mathrm{K}$-type supergiants have no measurements of this quantity. The B-type stars, even accounting for the $\sin i$ effect, are clearly rotating more slowly than the models predict. We could of course use models with a much lower initial rotational velocity, but we then loose the strong nitrogen enhancements. As Lennon et al. (2003) discussed, there are too many slow B-type rotators for this to be an aspect effect, these must be intrinsically slow rotators.

McErlean et al. (1999) and Dufton et al. (2000) discussed abundances in B-type supergiants in the Galaxy and the SMC. They also discussed the mismatch in predicted and observed effective temperatures and pointed out that the binary models could indeed produce nitrogen enriched B-type supergiants in the required temperature range. Wellstein et al. (2002) have produced an extensive grid of calculations for a range of initial conditions and indeed many of their systems do produce nitrogen enriched early B-type supergiants (in long period orbits with a remnant of the mass donor). They can also produce blue straggler stars on the main sequence, as is observed in the H-R diagram of NGC 330. Although these systems are still binaries, the predicted velocity amplitudes, 10 - 
$20 \mathrm{~km} \mathrm{~s}^{-1}$, and periods, $50-100 \mathrm{~d}$, make it unlikely that we would have detected these systems in our data. One problem which this scenario has in common with the rotating models is that the predicted rotational velocities are also too high as the accreting star (which in this picture is now the B-type supergiant) is expected to be spun up.

\section{Summary}

The early B-type giant and supergiant stars in NGC 330 are all nitrogen enriched by approximately a factor of 10 relative to the SMC pristine nitrogen abundance. Models which include the effects of rotationally induced mixing can explain the range of nitrogen abundances found in these stars, and in SMC Atype supergiants. However these models do not reproduce the range of effective temperatures occupied by the B-type stars, although they account well for the A-type supergiants. Models of binary evolution can produce nitrogen enriched giants and supergiants, as well as blue straggler stars, also seen in NGC 330. Nevertheless, neither set of models can reproduce the observed rotational velocities of the B-type stars, which for the NGC 330 sample are much too small compared to theory, particularly for stars near the end of main seqeuence. The mean nitrogen abundances in the K-type supergiants of NGC 330 are in good agreement with the B-star results, but the range of observed abundances is much smaller, which is difficult to reconcile if they are related groups of stars. In conclusion, the B-type stars considered here are still something of a puzzle and not well modeled by current stellar evolution calculations. Moreover, given the large number of these objects in NGC 330 it is clear that they are not the product of some peculiar evolutionary path and therefore represent an important challenge to current stellar evolution theory.

Acknowledgments. DJL would like to thank Norbert Langer, Alexander Heger, Philip Dufton and Cian Crowley for their contributions to this paper, and NOVA for funding a research visit to Utrecht during the course of which some of this work was completed.

\section{References}

Arp, H. 1959, AJ 64, 254

Dufton, P.L., McErlean, N.D., Lennon, D.J., Ryans, R.S.I. 2000, A\&A 353, 311

Grebel, E.K., James Roberts, W., Brandner, W. 1996, A\&A 311, 470

Heger, A., Langer, N. 2000, ApJ 544, 1016

Hill, V. 1999, A\&A 345,430

Langer, N., Maeder, A. 1995, A\&A 295, 685

Lennon, D.J., Mazzali, P.A., Pasian, F., Bonifacio, P., Castellani, V. 1994, Space Sci. Reviews 66, 169

Lennon, D.J., Dufton, P.L., Mazzali, P.A., Pasian, F., Marconi, G. 1996, A\&A 314, 243

Lennon, D.J., Dufton, P.L., Crowley, C. 2003, A\&A 398, 455

Maeder, A., Meynet, G. 2001, A\&A 373, 555

McErlean, N.D., Lennon, D.J., Dufton, P.L. 1999, A\&A 349, 553

Meynet, D., Maeder, G. 2000, A\&A 361, 101

Venn, K.A. 1999, ApJ 518, 405 
Vrancken, M., Lennon, D.J., Dufton, P.L., Lambert, D.L. 2000, A\&A 358, 639

Wellstein, S., Langer, N., Braun, H. 2001, A\&A 369, 939

\section{Discussion}

EL EID: If you have heard my talk, the tracks you have used for massive stars do not explain the morphology of the NGC 330. You may try to use the calculations I have presented before drawing conclusion about rotational effects or binarities. Our calculations (evolutionary tracks) are basically able to explain the distributions of stars in the NGC 330.

LENNON: That's what I just said in my talk - the evolutionary tracks I use do not explain the cluster's H-R diagram. I would add that the color-magnitude diagram for NGC 330 which you showed in your talk contains yellow supergiants, which previous studies show are not cluster members but foreground stars, etc.

HERRERO: If there is a fraction of binaries among the B-type supergiants, shouldn't there be a fraction of them among the A-type supergiants? And a second question, it is true that the probability of having eight stars with low rotational velocities just by chance is very low, if collected from a sample of stars with randomly distributed rotational axes? What about the possibility that in this case they are not?

LENNON: To answer the second question; studies of the $v$ sini distribution of B-type stars and Be stars indicate that there is no evidence for alignment of rotational axes within the cluster. On the contrary, the Be star studies indicate that axes are randomly distributed. To answer the first question: the interacting binary models of Wellstein et al. (2001) do not produce A-type supergiants, these are only produced by single star models.

Moffat: If I remember correctly, it is $\chi$ Per and not $\mathrm{h}$ Per that contains all (most?) of the RSGs and even then (thanks, Roberta!) in the halo of $\chi$ Per. Yet you lumped $\mathrm{h}+\chi$ together as one, and with NGC 330 in the SMC you labelled them as 'key tests of stellar evolution'. Does this not cause some concern?

LENNON: Yes, $\mathrm{h}+\chi$ Per is a complex region, the question of cluster membership is complicated by the fact that one is looking towards the Perseus arm (thanks, Nolan). This is why I prefer to work with clusters in the Magellanic Clouds, where it is easier to determine membership.

MAEDER: The comparisons of observed $v \sin i$ and model predictions is essential, but up to now some observations point towards higher velocities compared to models while your point towards lower velocities. Taken at face value, your challenging results suggest a relatively faster braking compared to models with isotropic mass loss, and we may thus think of equatorial mass ejection and/or magnetic coupling to be responsible for the differences.

SONNEBORN: The comparison of observations with evolutionary tracks $\left(L, T_{\text {eff }}\right)$ needs to consider the effect inclination for rapid rotators. A star with $v_{\mathrm{eq}}=300 \mathrm{~km} \mathrm{~s}^{-1}$ will be in different $L, T_{\text {eff }}$ places, depending on $i$. This is not significant for your stars since their $v \sin i$ are all low, but may be important for other stars. Appropriate model atmospheres for rotating stars for the Geneva tracks need to be generated to provide this inclination dependence. 
LENNON: I agree. For the fast rotators the H-R diagram needs to take this into account, as discussed by Grebel et al. (1996) for the B-type giants in this cluster.

VERDUGO: You showed around 8 to 10 A-type supergiants and a big dispersion of $\mathrm{N}$ abundances. What is the difference in luminosity between the most luminous A-type supergiants and the lowest luminous ones?

LEnNon: The A-type supergiants I show are from Kim Venn's (1999) paper. The luminosities cover a range similar to that of the B-type supergiants discussed here. I'm not sure if there is also a correlation between nitrogen abundance and luminosity for the A-type supergiants, but I should check this.

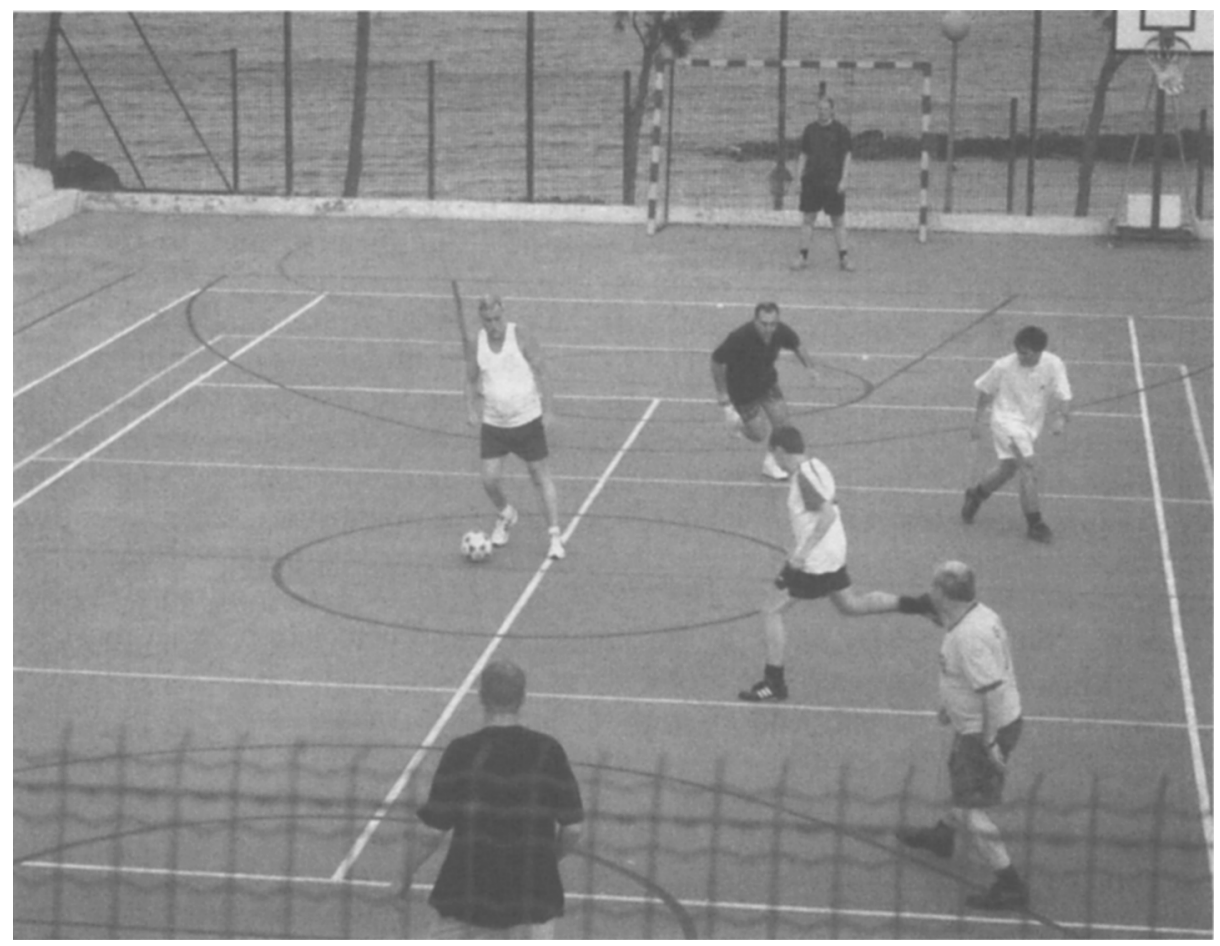

Some like it hot. From right: Danny Lennon, Hans Zinnecker, Dave Henley, Steve Smartt, Artemio Herrero, Jorick Vink, and Rolf-Peter Kudritzki 